\title{
Commentary
}

\section{Energy Source and Technology Options for Kenya: Towards Direct Conversion of Solar Energy to Mechanical Work}

\author{
Raphael Venson Makokha Otakwa ${ }^{1}$, Herick Othieno ${ }^{1}$, Andrew Odhiambo Oduor ${ }^{1}$, \\ Awange Joseph Lagat ${ }^{2}$ \\ ${ }^{1}$ Department of Physics and Materials Science, Maseno University, Kisumu, Kenya \\ ${ }^{2}$ Department of Spatial Sciences, Curtin University, Perth, Western Australia
}

Email address:

raphael.makokha@yahoo.com (R. V. M. Otakwa)

\section{To cite this article:}

Raphael Venson Makokha Otakwa, Herick Othieno, Andrew Odhiambo Oduor, Awange Joseph Lagat. Energy Source and Technology Options for Kenya: Towards Direct Conversion of Solar Energy to Mechanical Work. Advances in Applied Sciences. Vol. 2, No. 4, 2017, pp. 43-47. doi: 10.11648/j.aas.20170204.11

Received: May 7, 2017; Accepted: May 26, 2017; Published: July 7, 2017

\begin{abstract}
Sunlight though not heat, has inherent potential for direct conversion to mechanical work, just as heat does. The Physics pitching the possibility of direct conversion of nearly the entire sunlight received by a system into mechanical work has been formulated in this work. The feasibility of the concept has also been pursued, as well as alignment of the idea to the 2017-2027 predicted technology tipping points. Key to the ultimate realization of this propose, which argues for greater espousal of renewable energy options that foster attainment of engines for direct conversion of solar energy to mechanical work, are highly reflective and perfect mirrors. Smart areas and their associated favorable ecological footprints and climate change moderation will be among the key indicators of espousal of this conception.
\end{abstract}

Keywords: Solar Energy, Direct Conversion, Mechanical Work, Perfect Mirrors

\section{Introduction}

In Kenya, there exists laudable focus on how to support integrated approaches to addressing climate change in ways that embrace existing energy obligations and synergies, while minimizing trade-offs between climate change mitigation, adaptation and development [1]. This attention epitomizes the concept of clean compatible development (CCD) [2] that is entrenched in Kenya's energy policy framework; the National Action Plan on Climate Change (NAPCC), Vision 2030, the Constitution of Kenya, 2010, and the revised energy policy. The framework urges deeper reflection on, and formulation of energy access options that maximize the country's wealth of renewable energy sources that include, the 7000-10,000 megawatt (MW) geothermal energy potential [3] [4], high insolation rates, with an average of 5-7 peak sunshine hours and an average daily insolation of $4-6 \mathrm{kWh} / \mathrm{m}^{2}$ [5], as well as excellent wind regime areas that include Marsabit and Turkana in the northwest of the county and the edges of Rift Valley (which have average wind speeds of about $9 \mathrm{~ms}^{-1}$ at 50 metres) [6], 1,500 MW potential for large-scale hydro-electric power, with approximately 55 river sites suitable for small, mini and micro hydro-electric systems for rural electrification [7], and the country's agricultural activity that produces large amounts of agricultural wastes that can be used to produce electricity by implementing innovative biogas and biomass technologies [8]. Of these renewable energy sources, solar energy has been the most espoused; with the Kenyan photovoltaics (PV) market rated as one of the most successful off-grid markets in the developing world; often hailed as an exemplar of neoliberal development orthodoxy [9]. Solar thermal technologies for heating, cooking and drying have also been widely encouraged, albeit with not much aggregate data on their exploitation being available [10]. Solar energy studies in Kenya have generally focused on solar PV and solar thermal conversion pathways for solar energy to other energy forms.

Other noteworthy solar energy to electricity conversion options that have elicited growing interest include photogalvanics (PG) [11], photoemissives (PE) [12] and photomagnetics (PM) [13]. These can be expected to impel innovations within the corridor to the predicted technology tipping points: storage for all by 2018 ; robots and services by 
2021; internet of and for things, wearable internet, and three-dimension (3D) printing (manufacturing) by 2022; implantable technologies, big data for decisions, digital presence, and pocket supercomputers, by 2023; the connected home, 3D printing (human health), and ubiquitous computing, by 2025 ; 3D printing (consumer goods), the sharing economy, Artificial Intelligence (AI) and white collar jobs, by 2026; and driverless cars and smart areas, and AI-based decision-making, by 2027 [14].

The piezoelectric phenomenon, though not direct solar energy to electricity conversion option, is also gaining wide interest as an opportunity to scavenge energy from animal motion [15]. Thermal energy from the Sun can also be converted directly to electricity by means of thermoelectrics [16], ferroelectrics [17], magnetohydrodynamics [18], and electrogasdynamics [19]; clear opportunities through which Physics will contribute to the envisioned technological evolutions within the decade 2017-2027. In all these technologies, the iconic innovations will lie in improving the efficiency of the technologies' associated power systems, as well as use of locally available resources, so as to guarantee cost-effectiveness and affordability.

The option of converting solar energy directly to mechanical work through the thermodynamic pathway is proposed in this work. It is an area that holds immense potential, yet has received diminutive research interest, either from theoretical or practical perspectives. In this option, solar energy is converted to thermal energy that then performs shaft work through heat engines; an idea that can find use in the envisaged driverless cars, 3D printing, and robots and services This idea is underpinned by the principles of the Rankine Cycle [20], Stirling Cycle [21], or the Brayton Cycle [22], as well as shaft work. The shaft work through heat engines is essentially mechanical work that can be converted into electricity using an alternator. This is the idea that this paper seeks to introduce for attentiveness to.

It is essential to point out that mechanical work as used in this paper refers to the amount of energy transferred by a force. It is also necessary to emphasize that sunlight, in all solar thermal plants in which it is focused to heat a working fluid may seem to act as a hot reservoir, but on the whole, it is not heat, and should not be regarded as such. The basis for this assertion is that the more sunlight is concentrated in solar thermal plants, the more efficient they get in converting the sunlight to work. This argument may however differ in homogenous isotropic black body radiation, like inside an oven. The case for incoming rays from the Sun differs from this, as they are more or less aligned and distinct, hence, cannot be viewed as heat. In practical broad terms, heat is the randomly distributed energy within an object, which could be transmitted to a cooler object that is in contact with it.

\section{Theory}

Theoretically, the highest attainable efficiency in a system that converts sunlight to work is obtained when the sunlight is focused as much as possible, and used in a Carnot cycle [23].
In their article on "Photons as working body of solar engines," Laptev and Khlyap (2011) have articulated how absorbed radiant heat from the Sun is converted into work by Carnot cycles involving matter as a working body [24]. Laptev (2005) investigated the problem associated with enhancing efficiency in a system that converts sunlight to work by means of focusing the sunlight within a Carnot cycle framework under precise considerations of atmospheric effects, and raised attention to the second law of thermodynamics. Any extraction and conversion of heat (usually randomly distributed within an object), into some ordered energy (if it is to be used in an engine of thermally connected components), would thus be possible purely within the framework of the restrictions imposed by the second law of thermodynamics. The key issue about radiation from the Sun, as opposed to general heat is that radiation from the Sun is usually ordered, since it reaches the Earth approximately through a specified direction, and within a spherical angle of about $10^{-4}$ stredian (str), which limits its chances of disordering.

A keener scrutiny of the thermodynamics of solar cells, as investigated by Shockley and Queisser [25] reveals that sunlight does not really act as a heat resource in such devices. This contention aligns with recent literature about solar cells [26-28]. Indeed, multi-junction solar cells [29,30], as well as the bulk hetero-junction solar cells [31] are achieving efficiencies far beyond the Shockley-Queisser-established theoretical limit for single-junction cells [30], which affirms this viewpoint, thus demonstrating that more can be achieved from sunlight than is currently done. It is this line of thought that enthused consideration of pursuing the idea of conversion of solar energy directly into mechanical work. This idea is presented as a possible energy source and technology option for Kenya, in contribution to its dream of becoming an industrialized nation by the year 2030 [32]. The possibility of a direct and nearly complete conversion of aligned radiation into mechanical work is explained in this paper using a simple model, as well as through formulation of a supportive theory.

\section{Conceptualization/Methodology}

A number of aligned and initially monochromatic photons that are confined to move back and forth between two perfect reflecting mirrors; $\mathrm{M}_{1}$ and $\mathrm{M}_{2}$, placed a distance $x$ apart, as presented in Figure 1, formed the starting point of this conception.

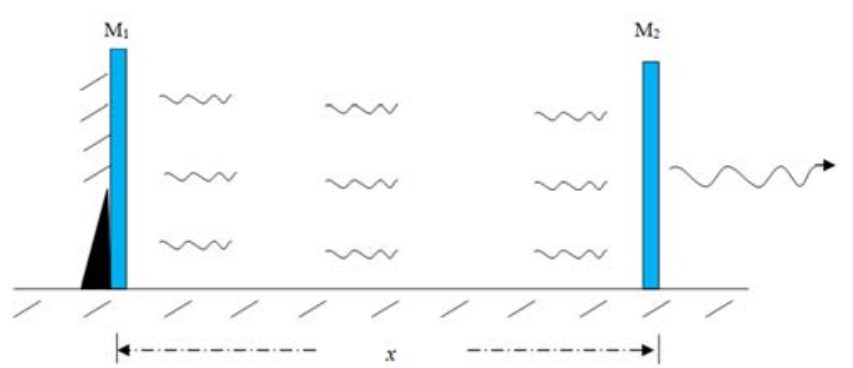

Figure 1. Diagram of the set-up, with the two perfect mirrors, $M_{1}$ and $M_{2}$ placed $x$ metres apart on a horizontal balance.

The perfect reflecting mirrors, as pictured in the model, 
referred to mirrors that reflect all light (and electromagnetic radiation in general) that is incident on them, without transmitting or absorbing it. The mirror on the left, i.e., $\mathrm{M}_{1}$ was fixed to a horizontal surface, while that one on the right, i.e., $\mathrm{M}_{2}$, was allowed to move - pushed right way by the force exerted by the photons between the mirrors. Friction was considered to be negligible between mirror $\mathrm{M}_{2}$ and the horizontal surface on which it slid. The mass of mirror $\mathrm{M}_{2}$, the initial distance between the mirrors, and the initial frequency associated with the photons were represented by $m, x_{0}$, and $f_{0}$, respectively. Observed changes in the velocity of mirror $\mathrm{M}_{2}$ were initially considered to be zero, thereafter, further changes were regarded to exclusively arise due to the successive elastic impacts of the photons on the mirrors' surfaces.

\section{The Formalism/Results}

Changes in the velocity of mirror $\mathrm{M}_{2}$ were evaluated by considering the principles of conservation of momentum and energy. Conservation of momentum requires that changes in the velocity of mirror $\mathrm{M}_{2} ; \delta v$ in (say) a time interval $\delta t$ obey the relation:

$$
m \delta v-N \frac{2 h f}{c} \times \frac{c \delta t}{2 x}=0
$$

where $2 h f / c$ is the change in momentum of each photon striking the surface of the mirror.

This change is ideally equal to $(h(2 f+\delta f)) / c$. However, it is well known that $\delta f \times \delta t$ is a second rank infinitesimal, and is therefore negligible. The factor $c \delta t / 2 x$ in equation (1) equals to the number of all such photon impacts on mirror $\mathrm{M}_{2}$ during time interval, $\delta t$, provided that $v \sim x / t \ll c$, for typical values of $v, x$, and $t$. From the principle of conservation of energy, it can be argued that:

$$
m \delta v=-N h \delta f
$$

Equation (2) provides the second equation in this formalism, which when solved from $t=0$ to some arbitrary time, $t_{a}$, results in:

$$
\frac{1}{2} m v^{2}=N h\left(f_{0}-f\right)
$$

Dividing the solution for momentum in equation (1) by $\delta t$, and rearranging, leads to:

$$
x \dot{v}=\frac{N}{m} h f
$$

Eliminating $f$ from Equations (3) and (4), leads to a differential equation for the kinematics of mirror $\mathrm{M}_{2}$ with respect to changes in its velocity with time as:

$$
x \dot{v}=\frac{N h f_{0}}{m}-\frac{1}{2} v^{2}
$$

Equation (5) can be solved numerically. However, it is apparent from its form that the velocity of the movable mirror $\left(\mathrm{M}_{2}\right)$ would approach its asymptotic (saturation) value; $v_{s} \equiv \sqrt{2 N h f_{0} / m}$, as $t$ gets larger and larger than a specific time scale (say), $\tau$.

Equation (5) can therefore be written as:

$$
x \dot{v}=\frac{1}{2}\left(v_{s}^{2}-v^{2}\right)
$$

Equation (5) can be re-written in a dimensionless form to enable or facilitate estimation of the magnitude of the time scale $\tau$. This can well be achieved by defining $\tau_{s}$ as:

$\tau_{s} \equiv \frac{x_{0}}{v_{s}}=x_{0} \sqrt{\frac{m}{2 N h f_{0}}}, \eta$ as $\eta \equiv t / \tau_{s}, \zeta$ as $\zeta \equiv x / x_{0}$, and $\beta \equiv v / v_{s}$, which leads to $\beta \equiv d \zeta / d \eta$.

Equation (5) can consequently be transformed into its dimensionless form as:

$$
2 \varsigma \frac{d \beta}{d \eta}=1-\beta^{2}
$$

The dimensionless form of Equation (5) consigns the saturation time scale $\tau$ to $\eta=1$ or, equivalently, to $t=\tau_{s}$, such that:

$$
t=\tau_{s} \equiv x_{0} \sqrt{\frac{m}{2 N h f_{0}}}
$$

Figure 2 is an illustration of the numerical solution to Equation (7) for $\zeta_{0} \equiv \zeta(0)=1$ and $\beta_{0} \equiv \beta(0)=0$. As can be observed from the figure, the moveable mirror, $\mathrm{M}_{2}$, reaches 0.45 of its saturation velocity; $v_{s} \equiv \sqrt{2 N h f_{0} / m}$ at $\eta=1$, or equivalently at $t=\tau_{s} \equiv x_{0} \sqrt{\frac{m}{2 N h f_{0}}}$. The figure illustrates the confirmation of solutions to Equation (7) for $\zeta_{0} \equiv \zeta(0)=1$, and $\beta_{0} \equiv \beta(0)=0$.

Meanwhile, according to equation (8), if $N h f_{0} \ll m c^{2}$, then the assumption $x_{0} / \tau_{s} \ll c$ that was made during the derivation of Equation (1) stands justified.

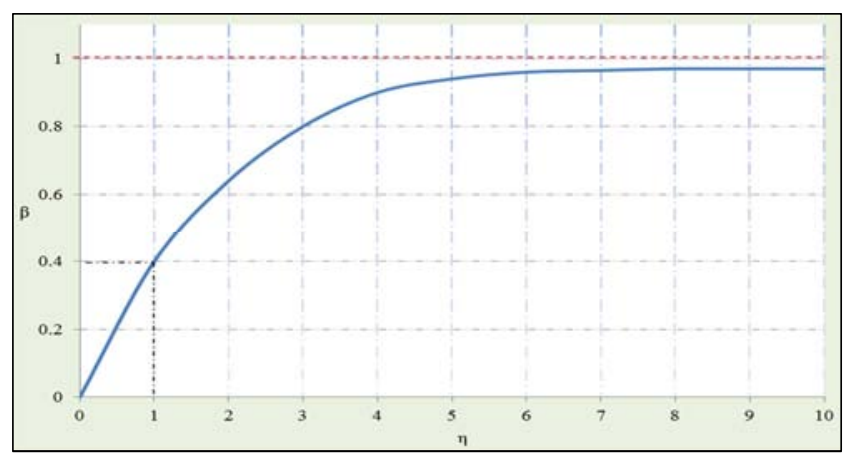

Figure 2. An illustration of the numerical solution to equation (7) for $\zeta_{0} \equiv \zeta(0)=1$ and $\beta_{0} \equiv \beta(0)=0$.

Considering the time scale, $\tau$ from a somewhat different perspective, i.e., seeing it as the time by which the total energy of all the photons active in the system is significantly transferred onto mirror $\mathrm{M}_{2}, \tau$ can be estimated by considering the total number of impacts made constantly by the photons onto mirror $\mathrm{M}_{2}$ until they lose a significant part of their initial energy. First of all, the total number of all such impacts can be considered to be of the order of $v_{s} / \delta v_{\text {ind }}$, where $\delta v_{\text {ind }}$ represents the mirror's typical velocity changes per impact due to each individual photon. The principle of conservation of momentum requires $m \delta v_{\text {ind }}$ to be of the order $2 h f_{0} / c$, so that $\delta v_{\text {ind }} \sim 2 h f_{0} / m c$. Consequently, the total number of successive impacts of the photons before entirely losing their 
initial energy will be:

$$
N_{\text {tot }} \sim \frac{v_{S}}{\delta v_{\text {ind }}} \sim \frac{\sqrt{2 N h f_{0} / m}}{2 h f_{0} / m c}=\sqrt{N m c^{2} / 2 h f_{0}},
$$

where we used $v_{s}=\sqrt{2 N h f_{0} / m}$.

Therefore, the total number of impacts per each individual photon will be:

$$
N_{\text {ind }}=\frac{N_{\text {tot }}}{N} \sim \sqrt{m c^{2} / 2 N h f_{0}}
$$

This means that $\tau$ by which the energy of all photons in the system is transferred onto mirror $\mathrm{M}_{2}$ will be about $\left(\frac{x_{0}}{c}\right) N_{\text {ind }}$ or $\tau \sim x_{0} \sqrt{\frac{m}{2 N h f_{0}}}$. This aligns with Equation (8).

\section{Conclusion and Recommendations}

The time scale for photons from the Sun's rays (which are of usually of typical energy $0.1 \mathrm{eV}$ ) held captive inside a device of length, $x \sim 0.3 \mathrm{~m}$ that includes a tiny moveable mirror of (say) mass $10^{-3} \mathrm{~kg}$, will be in the order of $10^{8} / \sqrt{N}$ seconds. Assuming that the total photon number $N$ is sufficiently large, $\tau$ will turn out to be extremely small. Therefore, the smallness of $\tau$, which is a necessary condition for such a solar energy to mechanical work conversion system to be practicable, has been satisfied by the presented formalism. Indeed, the test on which the formalism was underpinned is not an engine, as the involved processes do not provide a closed loop. Nonetheless, it serves as an introduction to the basis for the idea. Further exploration of the concept is therefore recommended, which exploration should ensure proviso for a closed loop that aligns with the reality for engines. The formalism demonstrates that innovations that Kenya, as well as areas that form the sunshine-rich part of the world should focus on for greater utilization of their abundant sunshine ought to venture into uncharted territories, like the direct conversion of solar energy to mechanical work, and not just the traditional lighting and heating applications. It is in such 'out-of-the-box' disruptive thinking and innovations that the areas can contribute effectively to the rapidly evolving techno-sphere, including positioning themselves to be the smart areas of the future, as foreseen through the lenses of the technology tipping points over the 2017-2027 decade [14]. Improved ecological footprints and climate change mitigation will be among the key indirect benefits resulting from such innovations.

\section{References}

[1] Peter Newell, Jon Phillips, Ana Pueyo, Edith Kirumba, Nicolas Ozor, Kevin Urama. The political economy of low carbon economy in Kenya. Institute of Development Studies (IDS), Working Paper 445, 2014.

[2] Lindsay C. Stringer, Andrew J. Dougill, Jen C. Dyer, Katherine Vincent, Florian Fritzsche, Julia Leventon, Mario Paulo Falcảo, Pascal Manyakaidze, Stephen Syampungani, Phillip Powell, Gabriel Kabala. Advancing climate compatible development:
Lessons from Southern Africa. Regional Environmental Change, April 2014, Volume 14, Issue 2, pp. 713-725.

[3] Republic of Kenya. Scaling up renewable energy program (SREP). Inter-sessional meeting of the SREP committee, Washington, D. C., September 8, 2011.

[4] Cyril Beland. Hot rocks: Kenya taps geothermal heat to boost power. http://phys.org/news/2016-03-hot-kenya-geothermal-bo ost-power.html, March 16, 2016. Accessed on May 23, 2017.

[5] Africa-EU Renewable Energy Cooperation Programme (RECP). Kenya renewable energy potential. http://www.africa-eu-renewables.org/market-informatio $\mathrm{n} /$ kenya/renewable-energy-potential, undated. Accessed on March 23, 2017.

[6] Maulidi Barasa. Wind regime analysis and reserve estimates (case study:-Kenya). Thesis for Master of Science in Energy Management, Department of Mechanical and Manufacturing Engineering, University of Nairobi, 2013.

[7] Kiplagat J. K., Wang R. Z., Li T. X. Renewable energy in Kenya: Resource potential and status of exploitation. Renewable and Sustainable Energy Reviews, 2011, Volume 12, pp. 2960-2973.

[8] Elmar Dimpl, Michael Blunck. Small-scale electricity generation from biomass: Experience with small-scale technologies for basic energy supply-Biogas. GTZ-HERA Poverty-Oriented Basic Energy Service, 2010.

[9] Rob Byrne, Kennedy Mbeva. The political economy of state-led transformations in pro-poor low carbon energy: A case study of solar PV in Kenya. Institute of Development Studies (IDS), STEPS Working Paper 91, Brighton: STEPS Centre, 2017.

[10] Stephen Karekezi. Renewable in Africa - meeting the energy needs of the poor. Energy Policy, September 2002, Volume 30, Issue 11-12, pp. 909-1144.

[11] Prerna Gangoti, Pooran Koli. Study of the enhancement on photogalvanics: Solar energy conversion and storage in EDTA-safranine O-NaLs system. Sustainable Energy Fuels, 2017, DOI: 10.1039/C7SE00083A.

[12] Alfred H. Sommer. Brief history of photo-emissive materials. Proceedings of SPIE 2022, photo-detectors and power meters, October 15, 1993, Volume 2.

[13] W.M. Fisher, S. C. Rand. Optically-induced charge separation and terahertz emission in unbiased dielectrics. Journal of Applied Physics, 2011, Volume 109, Issue 129901.

[14] Global Agenda Council on the Future of Software and Society. (2015). Deep shift: Technology tipping points and societal impact. Switzerland: World Economic Forum

[15] Qiang Zheng, Bojing Shi, Zhou Li, Zhou Li, Zhong Lin Wang. Recent progress on piezoelectric and tribo-electric energy harvesters in biomedical systems. Advanced Science, March 27, 2017, DOI: $10.1002 /$ advs.20170029.

[16] Xiao Zhang, Li-Dong Zhao. Thermoelectric materials: Energy conversion between heat and electricity. Journal of Materiomics, June 2015, Volume 1, Issue 2, pp. 92-105.

[17] V. A. Volpyas, A. B. Kozyrev, O. I. Soldatenkov, E. R. Tepina. Efficiency of thermoelectric conversion in ferroelectric film capacitive structures. Technical Physics, June 2012, Volume 57, Issue 6, pp. 792-796. 
[18] Ajith Krishnan R., Jinshah B.S. Magneto-hydrodynamic power generation. International Journal of Scientific and Research Publications, June 2013, Volume 3, Issue 6, pp. 1-11.

[19] Ajimotokan Habeeb A. A study of the trilateral flash cycles for low grade waste heat recovery - to power generation. PhD Thesis for the School of Engineering, Cranfield University, July 2014

[20] Sylvain Quoilin, Vincent Lemort. The organic Rankine cycle: Thermodynamics, applications and optimization. Encyclopedia of Life Support Systems (EOLSS). http://www.eols.net/Sample-Chapter/C05/E6-35-43-00. pdf. Accessed on May 22, 2017.

[21] Koichi Hirata. Schmidt theory for Stirling engines. National Maritime Research Institute. http://www.nmri.go.jp/eng/khirata/stirling/schmidt/sch midt_e.pdf. Accessed on May 23, 2017.

[22] B. Zohuri. Combine cycle driven efficiency for next generation nuclear power plants. Springer International Publishing, Switzerland, 2015.

[23] H.

Gichungi, http:/www.sv.uio.no/iss/english/research/projects/solartransitions/announcements/Kenya-Henry_Gichungi.pdf (Accessed on 10 November 2015).

[24] V. I. Laptev and H. Khlyap (2011). Photons as Working Body of Solar Engines, Solar Cells - New Aspects and Solutions, Prof. Leonid A. Kosyachenko (Ed.), ISBN: 978-953-307-761-1, In Tech, Available from: http://www.intechopen.com/books/solar-cells-new-aspe cts-and-solutions/photons-as-working-body-of-solarengi nes.

[25] W. Shockley, H. J. Queisser. Detailed balance limit efficiency of p-n junction solar cells. Journal of Applied Physics, 1961, Volume 32, pp. 510-520.

[26] Nathan S. Lewis, Daniel G. Nocera. Powering the planet: Chemical challenges in solar energy utilization. Proceedings of the National Academy of Sciences of the United States of America, 2006, Volume 103, Issue 43, pp. 15729-15735.

[27] International Energy Agency (IEA). Technology roadmap: Solar photovoltaic energy, 2014 http://www.iea.org/publications/freepublications/publications/ TechnologyRoadmapSolarPhotovoltaicEnergy_2014edition.pd f. Accessed on May 23, 2017.

[28] S. Aziz, S. Hassan. On improving the efficiency of a solar panel tracking system. Procedia Manufacturing, 2017, Volume 7, pp. 218-224.

[29] S. Kurtz, J. Geisz. Multijunction solar cells for conversion of concentrated sunlight to electricity. Optics Express, April 2010, Volume 18, Issue S1, pp. A73-78.

[30] Martin Green, Keith A. Emery, Yoshiro Hishikawa, Anita Ho-Baillie. Solar efficiency tables (version 49). Progress in Photovoltaics Research and Applications, November 2016, Volume 25, Issue 1.

[31] Jinyan Zhang, Cao Yu, Miao Yang, Gangqiang Dong, Zhikal Yi, Wei Long, Shihu Lan, Yue Zhang, Jingjing He, Minying Do, Xixiang Xu. Recent development progress of high efficiency emitter heterojunction solar cells. China Semiconductor Technology International Conference (CSTIC), 13-14 March 2016, pp. 1-18.

[32] Government of the Republic of Kenya, Ministry of Planning and National Development and the National Economic and Social Council (NESC), Office of the President. Kenya: Vision 2030, Government Printers, 2007, pp.1-136. 